\title{
Influence of primary beam energy on localized surface plasmon resonances mapping by STEM-EELS
}

Michal Horák ${ }^{1}$ and Tomáš Šikola ${ }^{2}$

${ }^{1}$ Brno University of Technology, Brno, Not Applicable, Czech Republic, ${ }^{2}$ Brno University of Technology, United States

Localized surface plasmon resonances (LSPR) are self-sustained collective oscillations of free electrons in metal nano- and microstructures. Mapping of LSPR with high spatial and energy resolution is necessary to understand their origin and properties. Scanning transmission electron microscopy (STEM) combined with electron energy loss spectroscopy (EELS) has become a standard technique to map LSPR with a nanometer spatial and $10 \mathrm{meV}$ to $100 \mathrm{meV}$ energy resolution over the last 15 years. In EELS, a swift electron propagating with a certain velocity loses a tiny part of its kinetic energy by performing work against the electric field produced by itself. Consequently, the loss probability measured by EELS is related to the induced electric field parallel with the electron beam. Despite that many works dealing with EELS measurement of LSPR have been published, there has been no experimental work discussing the experimental conditions during the measurement. Therefore, we have experimentally studied the influence of the primary beam energy and the collection semi-angle on the localized surface plasmon resonances measurement by STEM-EELS to make an instructive overview for the beginners in the field [1].

We have discussed the impact on experimental characteristics which are important to detect localized surface plasmon peaks in EELS successfully, namely: the intensity of plasmonic signal, the signal to background ratio, and the signal to zero-loss peak ratio considering a limited dynamic range of the spectrometer camera [1]. The plasmonic object of our interest were gold nanorods $(240 \mathrm{~nm} \times 80 \mathrm{~nm} \times 30$ $\mathrm{nm}$ ) fabricated by focused ion beam milling [2] of a $30 \mathrm{~nm}$ thick polycrystalline gold layer deposited on $30 \mathrm{~nm}$ thick silicon nitride membrane. We note that nanorods manufactured from the polycrystalline layer have a similar plasmonic quality as nanorods manufactured from the monocrystalline gold platelets [3]. We took a series of 3 rods and do the STEM-EELS measurement at the primary beam energy of $300 \mathrm{keV}$, $120 \mathrm{keV}$, and $60 \mathrm{keV}$ while keeping the convergence semi-angle at $10 \mathrm{mrad}$ and the collection semi-angle constant at $20.5 \mathrm{mrad}$. Figure 1(a) shows EEL spectra of one rod recorded at different beam energies. We clearly see that the signal corresponding to the LSPR is the strongest for the $60 \mathrm{keV}$ electron beam and the weakest for the $300 \mathrm{keV}$ electron beam. Moreover, if we consider the second peak in experimental EEL spectra in Figure 1(a) at $1.76 \mathrm{eV}$ corresponding to the longitudinal quadrupole mode, we see that lower beam energies are better for observation of weaker plasmon modes. If we consider measured raw EEL spectra in Figure 1(a), we clearly see that the peak at $1.08 \mathrm{eV}$ corresponding to the longitudinal dipole mode is the most noticeable when using $120 \mathrm{keV}$ electron beam. In the case of $300 \mathrm{keV}$ electron beam the background is enhanced by relativistic effects like the Čerenkov radiation as the speed of the $300 \mathrm{keV}$ electron is higher than the speed of the light in the silicon nitride membrane [4] with the refractive index around 2. On the other hand, the raw EEL spectra measured with a $60 \mathrm{keV}$ electron beam has the highest background in the lower energy loss region. This is caused by a higher probability of all scattering events as the mean free path of slower electrons in the sample is smaller. Focusing on EEL maps in Figure 2(a) we easily see that the EEL maps recorded at $60 \mathrm{keV}$ are rather noisy and the spatial distribution of the LSPR seems to be more confined when measuring with lower beam energies. However, inspecting the line profiles in Figure 2(b) we clearly see that the spatial distribution of the LSPR is similar for all three 
primary beam energies. Consequently, the only difference in the EEL maps is in the signal to noise ratio. Therefore, the optimal primary beam energy should be high enough to measure the signal at positions in the metal with a good signal to noise ratio.

To conclude, the best results in terms of the best signal-to-background ratio are obtained using a medium primary beam energy, in our case $120 \mathrm{keV}$. The primary beam energy should be high enough to suppress the scattering in the sample and at the same time should be low enough to avoid the appearance of relativistic effects. In the case of too high primary beam energy, for example $300 \mathrm{keV}$, the relativistic effects in the supporting membrane play a non-negligible role and lead to a higher intensity of the background. However, the advantage of the $300 \mathrm{keV}$ electron beam is a lower scattering probability resulting into a better signal to noise ratio in the case of spatial EEL maps of LSPR modes. We note that in the case of a better monochromatization of the primary electron beam (far below $0.1 \mathrm{eV}$ ), the elastic part of the background would be significantly reduced which should lead to much better signal to background ratio at lower primary beam energies.

Acknowledgements

Supported by MEYS CR under the project CzechNanoLab (LM2018110, 2020-2022) and Brno University of Technology (FSI-S-20-6485). M. H. acknowledges the support of Thermo Fisher Scientific and CSMS scholarship 2019.

(a) EEL spectra

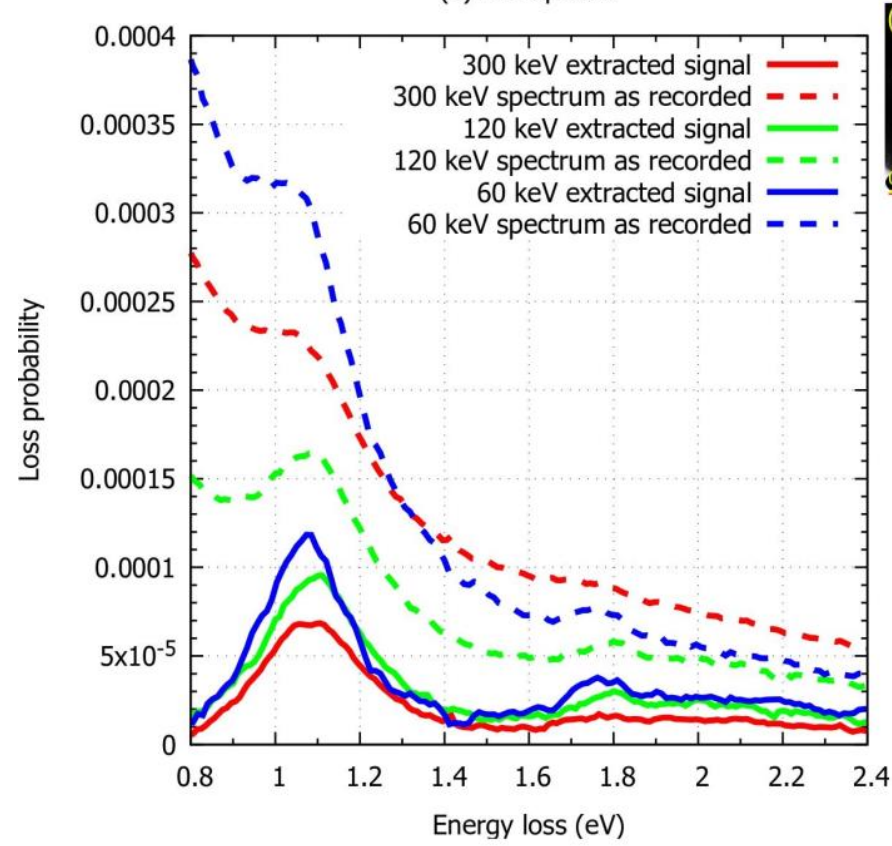

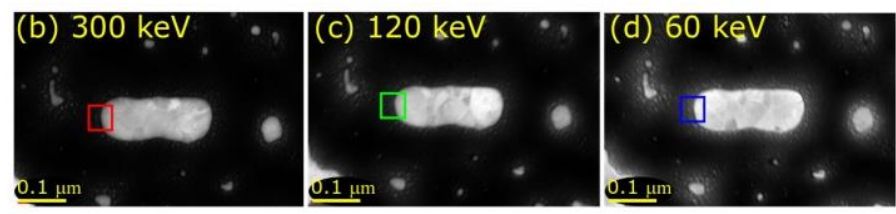

(e)

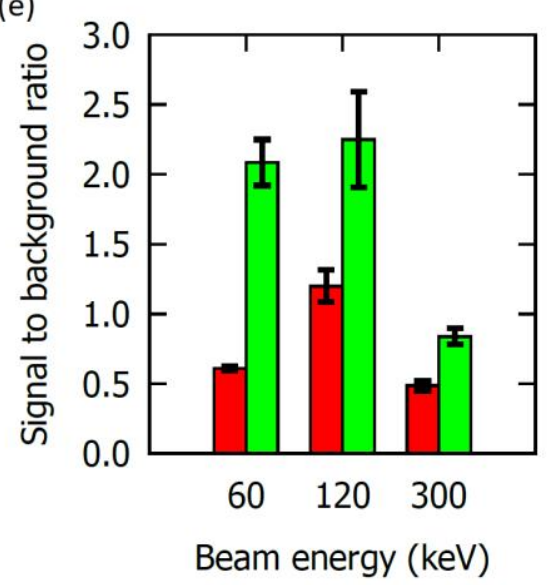

Figure 1. EEL spectra of the same rod at different beam energies: (a) measured raw EEL spectra and extracted signal; (b-d) STEM annular dark field (ADF) images of the rod with marked area for integration of EEL spectra in (a) recorded during STEM-EELS mapping at $300 \mathrm{keV}$ (b), $120 \mathrm{keV}$ (c), and $60 \mathrm{keV}$ (d); (e) signalto-background ratio for the longitudinal dipole (green) and quadrupole mode (red). 

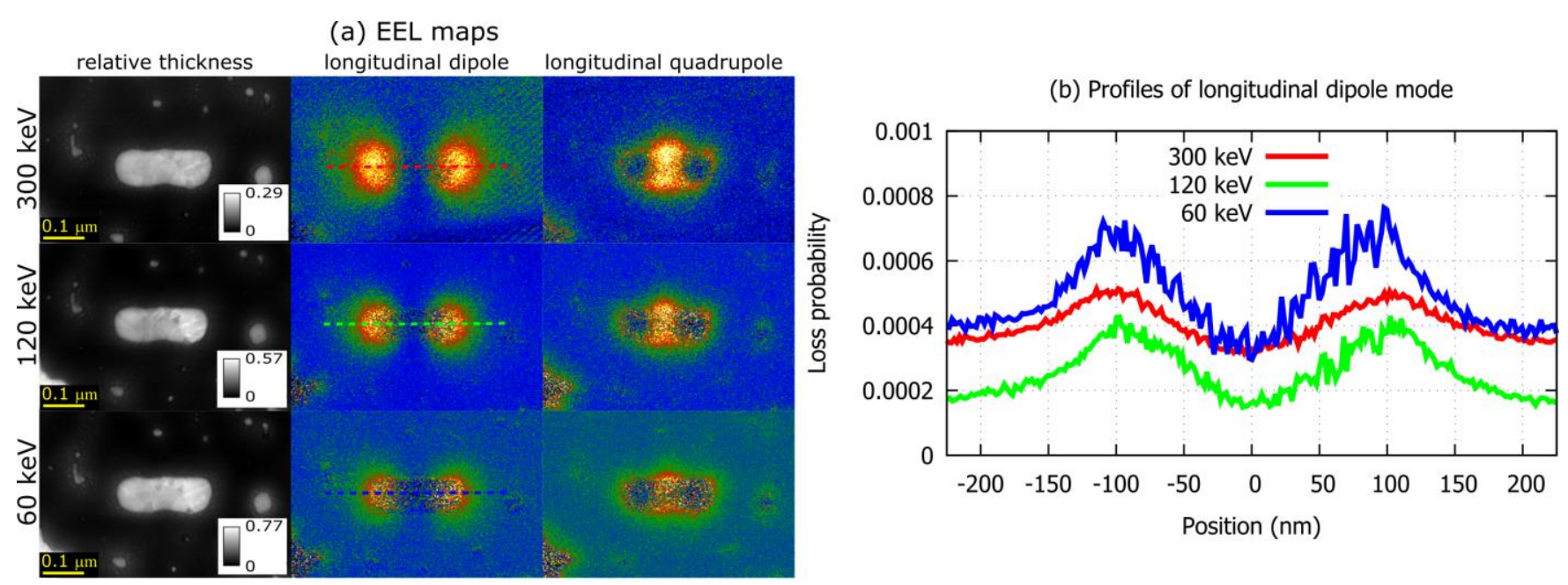

Figure 2. (a) Maps measured by EELS: relative thickness of the gold nanorod and EEL maps of the longitudinal dipole mode at $1.08 \mathrm{eV}$ and the longitudinal quadrupole mode at $1.76 \mathrm{eV}$ recorded at different primary beam energies $(300 \mathrm{keV}, 120 \mathrm{keV}$, and $60 \mathrm{keV})$. (b) Line profiles of the EEL maps of the longitudinal dipole mode along the dashed black lines in (a). The zero position corresponds to the middle of the rod.

\section{References}

[1] M. Horák, T. Šikola, Influence of experimental conditions on localized surface plasmon resonances measurement by electron energy loss spectroscopy, Ultramicroscopy 216 (2020) 113044.

[2] M. Horák, K. Bukvišová, V. Švarc, J. Jaskowiec, V. Křápek, T. Šikola, Comparative study of plasmonic antennas fabricated by electron beam and focused ion beam lithography, Sci. Rep. 8 (2018) 9640.

[3] L. Kejík, M. Horák, T. Šikola, V. Křápek, Structural and optical properties of monocrystalline and polycrystalline gold plasmonic nanorods, Opt. Express 28 (2020) 34960-34972.

[4] M. Horák, M. Stöger-Pollach, The Čerenkov limit of Si, GaAs and GaP in electron energy loss spectrometry, Ultramicroscopy 157 (2015) 73-78.

WIMRR 